\title{
A large-scale survey of genetic copy number variations among Han Chinese residing in Taiwan Chien-Hsing Lin ${ }^{1}$, Ling-Hui Li², Sheng-Feng $\mathrm{Ho}^{2}$, Tzu-Po Chuang2, Jer- Yuarn $\mathrm{Wu}^{2}$, Yuan-Tsong Chen ${ }^{2}$ and Cathy SJ Fann*1,2
}

\author{
Address: ${ }^{1}$ Department of Life Sciences and Institute of Genome Sciences, National Yang-Ming University, Taipei, Taiwan and ${ }^{2}$ Institute of \\ Biomedical Sciences, Academia Sinica, Taipei, Taiwan \\ Email: Chien-Hsing Lin - eviliori.tw@yahoo.com.tw; Ling-Hui Li - lli@ibms.sinica.edu.tw; Sheng-Feng Ho - philip@ibms.sinica.edu.tw; Tzu- \\ Po Chuang - clorence@gmail.com; Jer-YuarnWu - jywu@ibms.sinica.edu.tw; Yuan-Tsong Chen - chen0010@ibms.sinica.edu.tw; \\ Cathy SJ Fann* - csjfann@ibms.sinica.edu.tw \\ * Corresponding author
}

Published: 24 December 2008

BMC Genetics 2008, 9:92 doi:10.1/86/147/-2156-9-92
Received: 4 June 2008

Accepted: 24 December 2008

This article is available from: http://www.biomedcentral.com/I47I-2I56/9/92

(c) 2008 Lin et al; licensee BioMed Central Ltd.

This is an Open Access article distributed under the terms of the Creative Commons Attribution License (http://creativecommons.org/licenses/by/2.0), which permits unrestricted use, distribution, and reproduction in any medium, provided the original work is properly cited.

\begin{abstract}
Background: Copy number variations (CNVs) have recently been recognized as important structural variations in the human genome. CNVs can affect gene expression and thus may contribute to phenotypic differences. The copy number inferring tool (CNIT) is an effective hidden Markov model-based algorithm for estimating allele-specific copy number and predicting chromosomal alterations from single nucleotide polymorphism microarrays. The CNIT algorithm, which was constructed using data from 270 HapMap multi-ethnic individuals, was applied to identify CNVs from 300 unrelated Han Chinese individuals in Taiwan.

Results: Using stringent selection criteria, 230 regions with variable copy numbers were identified in the Han Chinese population; 133 (57.83\%) had been reported previously, 64 displayed greater than $1 \% \mathrm{CNV}$ allele frequency. The average size of the CNV regions was $322 \mathrm{~kb}$ (ranging from 1.48 $\mathrm{kb}$ to $5.68 \mathrm{Mb}$ ) and covered a total of $2.47 \%$ of the human genome. A total of 196 of the CNV regions were simple deletions and 27 were simple amplifications. There were 449 genes and 5 microRNAs within these CNV regions; some of these genes are known to be associated with diseases.

Conclusion: The identified CNVs are characteristic of the Han Chinese population and should be considered when genetic studies are conducted. The CNV distribution in the human genome is still poorly characterized, and there is much diversity among different ethnic populations.
\end{abstract}

\section{Background}

The human genome contains many DNA sequence variations, including single nucleotide polymorphisms (SNPs), short nucleotide insertions or deletions, tandem repeat sequences, and transposable elements [1]. Recent human genome studies have revealed that copy number variations (CNVs) are more common than previously thought. Some CNVs are associated with gene expression levels and thus may contribute to phenotypic differences [2-7]. Each CNV is a DNA segment $>1 \mathrm{~kb}$ that shows variation within the population in terms of a deletion and/or an amplification. Although SNPs are regarded as the main 
source of phenotypic differences among humans, CNVs also have a large impact on differential gene expression [2].

Microarray-based approaches, so called "molecular karyotyping", have been used to detect subtle chromosomal structure variations on the genome-wide scale [8]. SNP microarrays are high-resolution tools for genotyping that can be used to simultaneously detect copy number (CN) alterations and loss-of-heterozygosity $[9,10]$. The copy number inferring tool (CNIT), an algorithm recently developed for use with Affymetrix GeneChips, efficiently predicts regions with subtle $\mathrm{CN}$ changes and is based on a hidden Markov model (HMM) [11]. CNIT had higher accuracy and lower variation in $\mathrm{CN}$ estimation than other programs, including the copy number analysis tool [9] and the copy number analysis with regression and tree approach [12]. In this study, $100 \mathrm{~K}$ GeneChip intensity data from 270 HapMap multi-ethnic individuals were used to determine the parameters of the CNIT algorithm. Intensity data from 300 normal unrelated individuals were then used to predict $\mathrm{CNV}$ regions using CNIT.

Stringent selection criteria were used to classify true and false $\mathrm{CN}$-altered predictions: $\mathrm{CN}$-altered regions found in at least two individuals were classed as CNV regions. A total of 230 copy number-variable regions (CNVRs) were identified in the sample population, 64 of which $(27.83 \%)$ had a CNV allele frequency $\geq 1 \%$. Of these 230 CNVRs, $133(57.83 \%)$ had previously been reported in the genomic variant database http://projects.tcag.ca/varia tion/. The CNVRs ranged from $1.48 \mathrm{~kb}$ to $5.68 \mathrm{Mb}$ (mean $=322 \mathrm{~kb}$ ), and contained 449 genes and 5 microRNAs (miRNAs). Sixty-three CNVRs (27.39\%) were associated with segmental duplications (SDs), which are known to induce non-allelic homologous recombination and produce structural variations.

\section{Results}

\section{Detection of regions with copy number alterations}

In this study, 646 samples and 5 complicated processes were used to detect genetic regions with $\mathrm{CN}$ alterations. A flowchart of the study design is presented in Figure 1. First, the Affymetrix $100 \mathrm{~K}$ GeneChip intensity data from HapMap 270 multi-ethnic individuals were used to construct the CNIT algorithm. The intensity data from 376 unrelated Han Chinese individuals in Taiwan were used for $\mathrm{CNV}$ region identification. Although the SNP call rate was one of the quality control indexes for the GeneChip experiments, 76 of the 376 Han Chinese individuals $(20 \%)$ having larger numbers of $\mathrm{CN}$-altered regions were excluded from the following analyses. Using the CNIT algorithm, a total of $13,729 \mathrm{CN}$-altered events were predicted in the 300 individuals. Certain factors intrinsic to GeneChips are thought to affect probe intensity, so addi- tional criteria were used to eliminate false-positive results. For example, sequence variations within restrictionenzyme cutting sites or SNP probe sequences can directly alter SNP probe intensity and reduce the accuracy of $\mathrm{CN}$ estimation. Therefore, quantitative PCR (qPCR) experiments were used to validate more than 200 CNIT-predicted events from the first selection round and the results were analyzed. The following criteria were then used to eliminate false-positive $\mathrm{CN}$-altered findings: (1) the number of consecutive SNPs was required to be $\geq 6$ and $<$ 15 with $P \leq 0.05$; or (2) the number of consecutive SNPs was required to be $\geq 15$. Using these additional criteria, the false-positive rate (FPR) of the validated data was reduced from 0.3 to 0.005 ; however, $34.15 \%$ of true $\mathrm{CN}$ altered events were lost. After filter selection, a total of 4,288 CN-altered events in 549 genomic regions were retained. As an additional measure of cross-platform validation, results from Agilent $244 \mathrm{~K}$ comparative genomic hybridization (CGH) arrays from two selected individuals were compared to the results from the CNIT predictions and results were consistent between the two arrays. An example of one extra copy of a genomic segment of chromosome 21 is shown in Figure 2.

\section{Regions of copy number variants among Han Chinese population}

Among the 300 individuals, 76 and 224 DNA samples were extracted from peripheral blood and cell lines, respectively. It is known that subtle chromosomal abnormalities can be induced during cell culture. Although all these cell lines were freshly cultured, some chromosomal abnormalities might still have been generated during culture. To exclude this possibility, only those $\mathrm{CN}$-altered regions that were identified in more than two individuals were classed as CNVRs. Of the $549 \mathrm{CN}$-altered segments, 230 were classified as CNVRs in the Han Chinese population (Additional File 1). Among these 230 CNVRs, 133 $(57.83 \%)$ had been reported previously and 97 (42.17\%) were unique to this study. Ten of these novel CNVRs $(10 \%)$ were validated using qPCR. Of the 230 CNVRs, 196 $(85.22 \%)$ had simple deletion alleles, $27(11.74 \%)$ had simple amplification alleles, and 7 (3.04\%) were comprised of both deletion and amplification alleles. These CNVRs ranged from $1.48 \mathrm{~kb}$ to $5.68 \mathrm{Mb}($ mean $=322 \mathrm{~kb}$ ) and covered a total of $2.47 \%$ of the human genome. As shown in Figure 3, these CNVRs are widely dispersed throughout the genome, with the exception of chromosomes 20 and X. Moreover, 64 of the 230 CNVRs had a $\mathrm{CNV}$ allele frequency greater than $1 \%$. Of the CNVs in this study, $27 \%$ were associated with SDs, similar to previous results (24\%) [5].

The individuals in this study shared the same ancestry as HapMap individuals from Beijing, so the CNV regions identified among Han Chinese in Taiwan were compared 


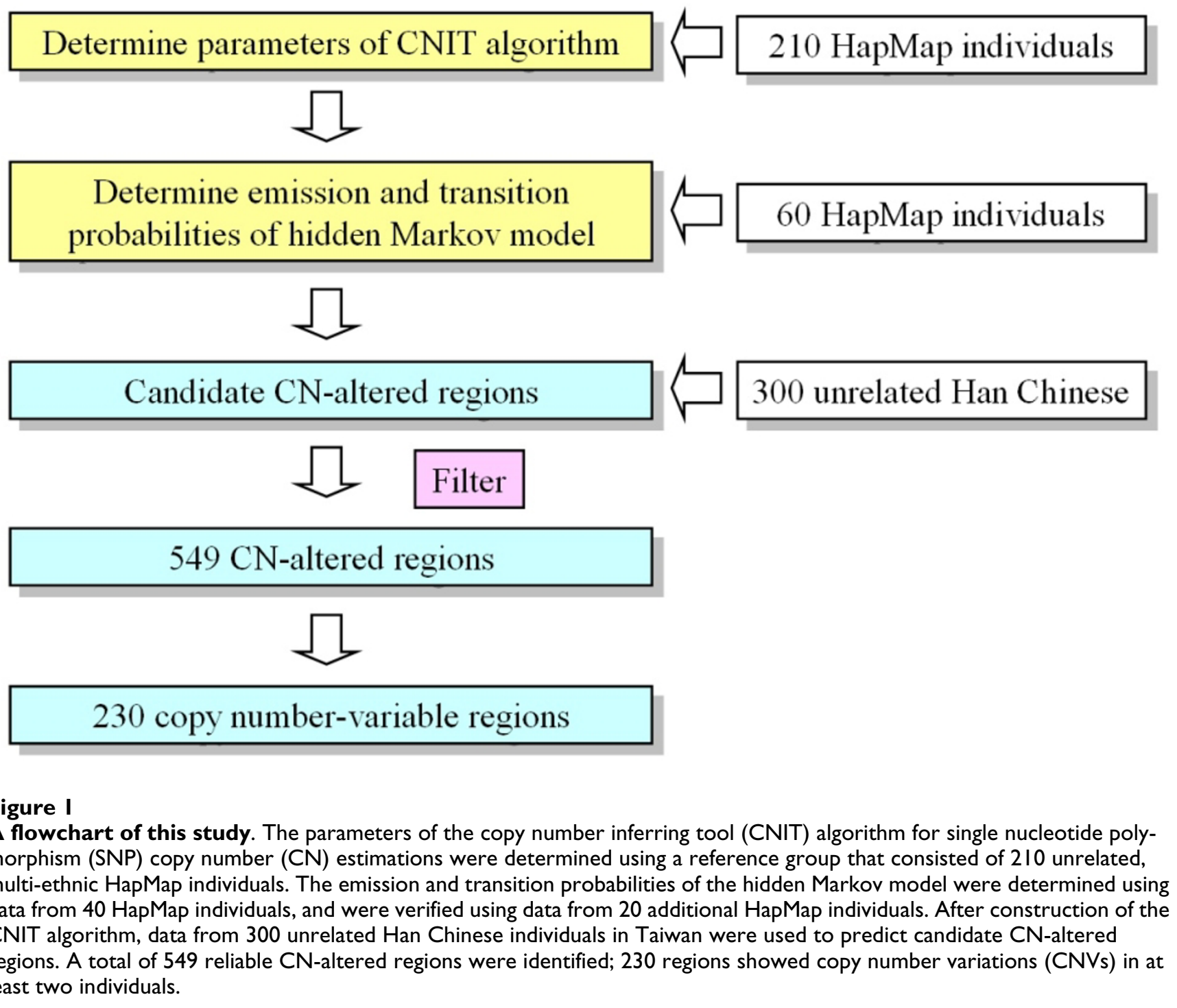

with those identified among Beijing residents [5]. Sixty $\mathrm{CNV}$ regions from the 45 unrelated HapMap Beijing individuals were detected using the $100 \mathrm{~K}$ GeneChip and CNIT algorithm, and $12(20 \%)$ of these were also observed in the samples of this study.

\section{Genes within copy number variation regions}

There were 449 genes located within 81 of the 230 CNVRs, and most of these genes $(56.35 \%)$ had simple amplifications (Additional File 2). The 449 genes identified are involved in many biological functions, including ion transport, metabolism, and cell-surface functions. Some of the identified genes are involved in potassium and/or sodium transport, such as the KCNT1, KCNJ8, SLC2A6 and SLC16A3 genes, and some are known to be involved in metabolism, such as the AMY genes that catalyze the first step in digestion of dietary starch and glyco- gen [13]. The copy numbers of the AMY1 gene are positively correlated with salivary amylase protein levels, which might result in differences in the digestion of starchy foods among individuals [14].

MicroRNAs (miRNAs) have recently been shown to be involved in the regulation of development and physiology in animals and plants [15]. miRNAs can specifically recognize target mRNAs with complementary sequences and cause translational repression or site-specific cleavage of the target. Five miRNAs in four CNVRs were identified in this study (Table 1) and all had previously been reported to have variable $\mathrm{CN}[5,7]$. Six target genes of these miRNAs had been predicted using the MIRANDA software http://www.microrna.org/, but none had been validated. Some $\mathrm{CN}$-variable genes have been shown to be correlated with diseases (Table 2), including the egl nine 


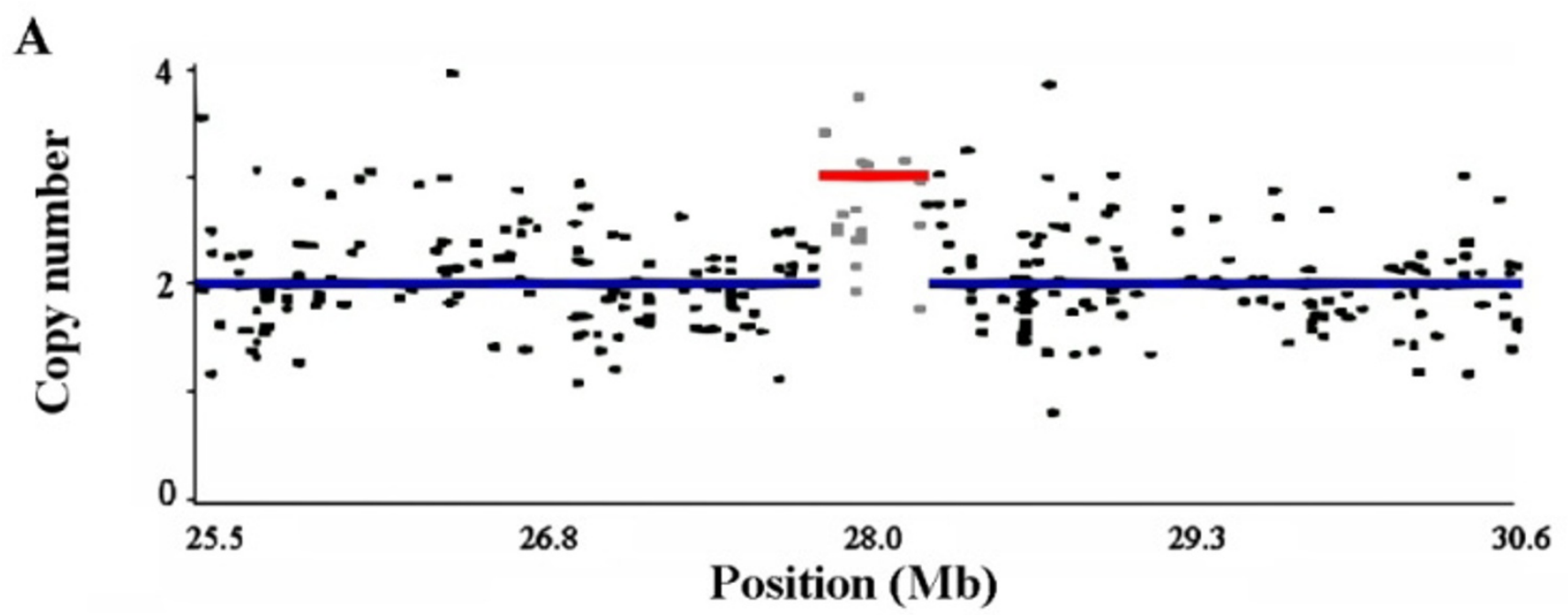

B

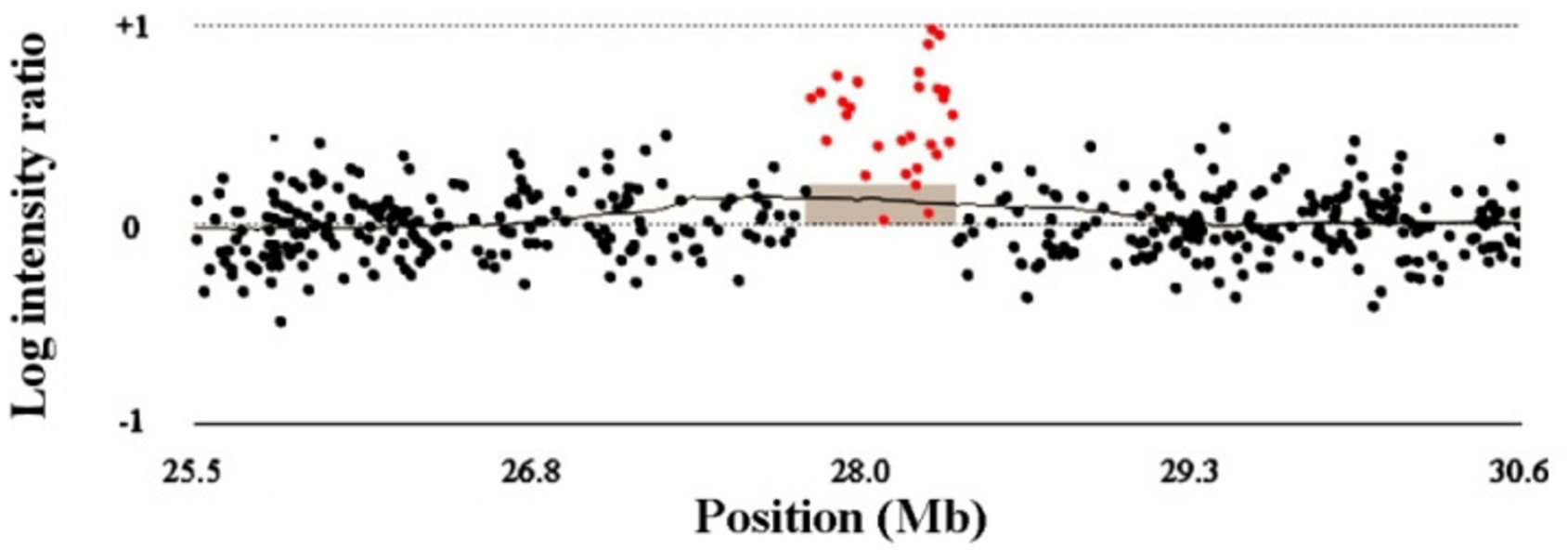

Figure 2

GeneChip and comparative genomic hybridization (CGH) array data from a Han Chinese individual revealing a single copy-number (CN)-altered segment on chromosome $2 \mathrm{I}$. (A) Affymetrix $100 \mathrm{~K}$ GeneChip analysis using the copy number inferring tool (CNIT). The size of the amplified segment (red) was $373 \mathrm{~kb}$. There were I7 SNPs within this region (mean $\mathrm{CN}=2.70$ ). (B) Agilent $244 \mathrm{~K} \mathrm{CGH}$ array analysis. The size of the amplified segment (shaded box) was $5 \mathrm{I} 2 \mathrm{~kb}$ based on a threshold z-score of 2.5 .

homolog 1 (EGLN1) and dopamine beta-hydroxylase $(D B H)$ genes. The EGLN1 gene is located on chromosome 1q42.1. Deficiency of this gene causes familial erythrocytosis type 3 (ECYT3) (MIM\# 609820), which is characterized by increased serum hemoglobin and hematocrit levels, but normal serum erythropoietin levels. Defects in the DBH gene cause DBH deficiency (MIM\# 223360), which is characterized by marked deficits in autonomic and cardiovascular function.

\section{Discussion}

In this report, a newly developed algorithm, CNIT, was used to identify CNVRs in a normal Han Chinese population. A key assumption of the CNIT algorithm construction is that the $\mathrm{CN}$ value of each SNP in the reference group should be two; however, this is not always realistic. Some SNPs with CN aberrations were excluded before constructing the CNIT algorithm due to the availability of $\mathrm{CNV}$ data from HapMap individuals. When these CNVs in 


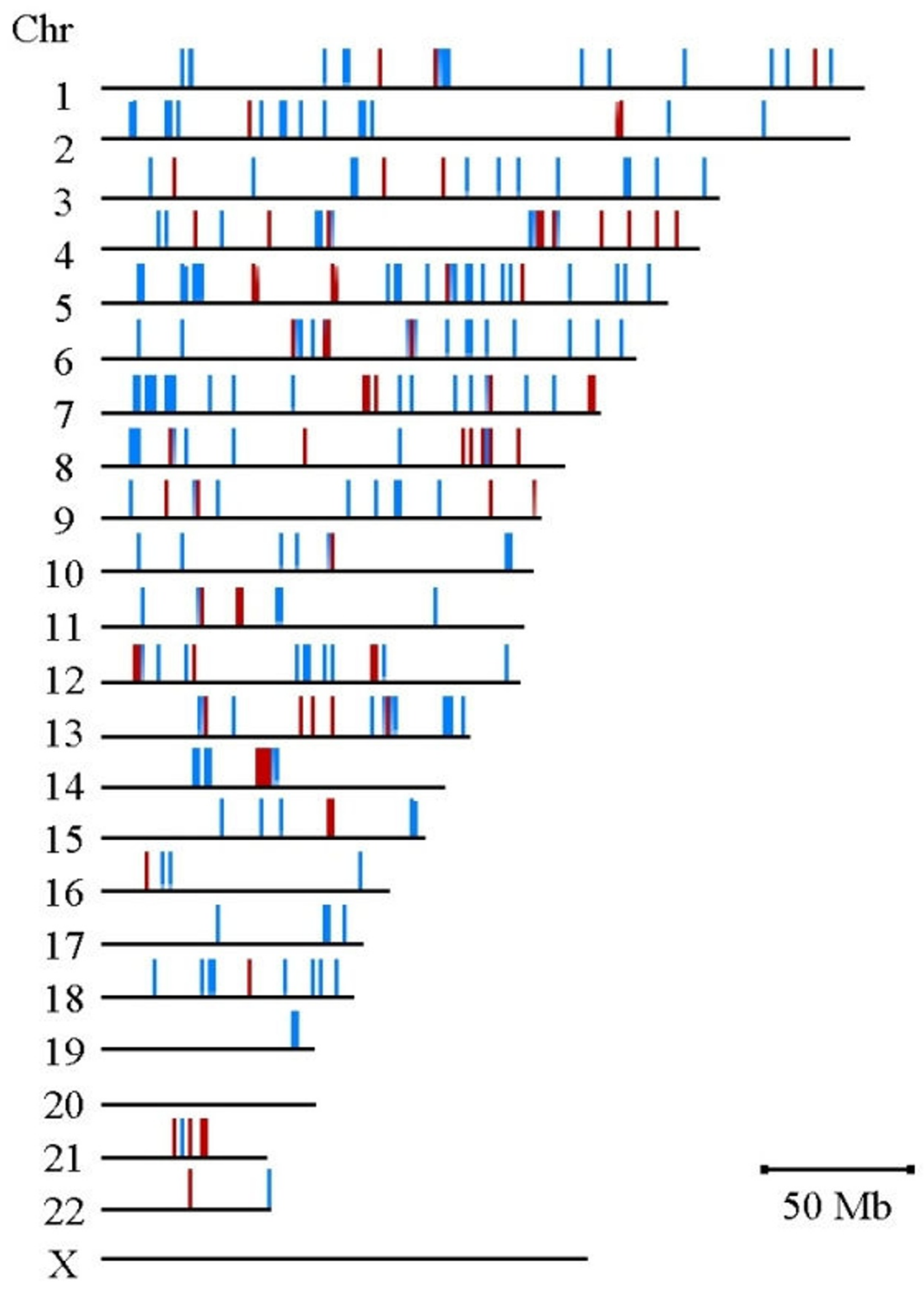

Figure 3

The copy number variation (CNV) map of a Han Chinese population in Taiwan. There were $230 \mathrm{CNV}$ regions (blue) identified from 300 unrelated individuals. Of these regions, 64 had greater than I\% CNV allele frequency and were classed as copy-number polymorphic regions (red). 
Table I: MicroRNAs in the copy number-variable regions (CNVRs)

\begin{tabular}{|c|c|c|c|c|c|c|}
\hline CNVR ID & Chr & Position (bp) & Variant Type & \# of observations ${ }^{a}$ & miRNA $^{b}$ & Target Gene ( $P$ value $)^{c}$ \\
\hline 102 & 7 & $3488259-5891682$ & Loss and Gain & 4 & hsa-mir-589 & \\
\hline 127 & 8 & |47|3230-|4862195 & Loss & 2 & hsa-mir-383 & SNCG $\left(9.71 \times 10^{-6}\right)$ \\
\hline 143 & 9 & $7236350 I-72631779$ & Loss & 2 & hsa-mir-204 & $\begin{array}{c}\operatorname{NELF}\left(1 \times 10^{-6}\right) \\
\operatorname{PLXDC2}\left(7.1 \times 10^{-6}\right) \\
\text { XYLTI }\left(1.5 \times 10^{-7}\right)\end{array}$ \\
\hline \multirow[t]{2}{*}{212} & 17 & $72497067-78181864$ & Gain & 5 & hsa-mir-338 & \\
\hline & & & & & hsa-mir-657 & BDKRB2 $\left(6.7 \times 10^{-7}\right) \mathrm{HOXBI} 3\left(7.1 \times 10^{-6}\right)$ \\
\hline
\end{tabular}

a Total number of gains and losses observed for a CNVR.

b MicroRNA overlapping a CNVR based on microRNAs of the UCSC March 2006 assembly.

c Target gene of microRNA based on MIRANDA software analysis using a I $\times 10^{-5}$ cut-off [19].

the reference group were excluded, the identification rate of known CNVs in the test group increased from $23.30 \%$ to $25.46 \%$. Owing to the stringent criteria in this study (that is, the parameters of the HMM method and the selection criteria), the false-negative rate (FNR) might be high, resulting in an underestimation of the CNV allele frequency. Nevertheless, these stringent criteria largely reduced the FPR and yielded reliable CNV results. Higher resolution microarrays or other technological approaches are needed to further address these structural variations in detail.

Most of the CNVRs (85.22\%) identified in this study were simple deletions; only a few were simple amplifications $(11.74 \%)$. The divergence might be due to the emission probability (EP) of the HMM method in the CNIT algorithm. The intensity distribution in a cell line with three copies of the $\mathrm{X}$ chromosomes was examined using $100 \mathrm{~K}$ GeneChip data and the CNIT algorithm. Intensity differences between samples containing gene amplifications $(\mathrm{CN}=3)$ and those containing normal $\mathrm{CNs}(\mathrm{CN}=2)$ were less distinct than the difference between samples containing hemizygous deletions $(\mathrm{CN}=1)$ and normal $\mathrm{CNs}(\mathrm{CN}$ estimations for one, two and three copies of $\mathrm{X}$ chromosomes were $1.38 \pm 0.42,2.08 \pm 0.47$ and $3.03 \pm 1.49$, respectively). To eliminate false-positive findings, a stringent EP was used for amplifications (the EP for amplifications was the reverse of the EP for deletions), and as a result, some CNVs with amplifications might have been lost in this CNV survey.

In this large-scale survey of CNVs in a Han Chinese population in Taiwan, 97 CNVRs (42.17\%) were unique; other CNVRs have previously been reported in Asian populations [5]. In the current genomic variant database, most CNVs are rare (allele frequency $<3 \%$ ). Fewer than 200 CNVRs had an allele frequency of $>5 \%$ and these CNVRs were detected using different microarray platforms such as Affymetrix $500 \mathrm{~K}$ GeneChips and CGH arrays. The probe distribution of the $100 \mathrm{~K}$ GeneChip data was quite differ- ent from the $500 \mathrm{~K}$ GeneChip or CGH array data. Therefore, it was difficult to precisely compare the results across different platforms. The reliability of the CNVRs found in this study was supported by stringent selection criteria, agreement with previous independent studies, qPCR analysis and CGH experiments.

Some $\mathrm{CN}$-variable genes identified in this study are known to be correlated with diseases, but individuals in the current study were healthy. Disease models and environmental factors are critical to disease etiology, perhaps explaining why the individuals with gene $\mathrm{CN}$ changes were healthy. The well-developed CNIT algorithm for 100 K GeneChip data can be used in future studies to identify subtle chromosome abnormalities http://www.csj fann.ibms.sinica.edu.tw/EAG/Program/CNIT/CNIT.htm.

\section{Conclusion}

CNVs have recently been recognized as an important structural variation in the human genome. In this study, $42.17 \%$ of the identified CNVRs were novel, indicating that the CNV loci of the human genome are still not fully understood and that there is much diversity among different ethnic populations. Most CNV allele frequencies were low, and only two CNVRs had a frequency greater than $10 \%$. This observation is consistent with results reported previously by Jakobsson et al [4]. The CNVs reported in this study are characteristic of Han Chinese populations and should be considered when genetic studies are conducted.

\section{Methods}

\section{SNP microarray intensity data}

Data from 376 unrelated individuals were randomly collected from the Taiwanese Cell and Genome Bank [16]; the raw microarray intensity data have been described in previous studies $[11,17]$. Individual genotyping was performed by the National Genotyping Center (Academia Sinica, Taipei, Taiwan) using the Affymetrix $100 \mathrm{~K}$ GeneChip Human Mapping set (Affymetrix, Santa Clara, CA, 
Table 2: Genes in the copy number-variable regions (CNVRs) are associated with disease and disease susceptibility

\begin{tabular}{|c|c|c|c|c|c|c|}
\hline CNVR ID & Chr & Position (bp) & Variant Type & \# of observations ${ }^{b}$ & Gene symbolc & Disease $^{d}$ \\
\hline \multirow[t]{3}{*}{$I^{a}$} & I & $|73| 344|-1964| 958$ & Gain & 4 & $A L D H 4 A I$ & Hyperprolinemia, type II \\
\hline & & & & & MRT4 & Mental retardation \\
\hline & & & & & PAX7 & Rhabdomyosarcoma, alveolar \\
\hline \multirow[t]{2}{*}{$13^{a}$} & 1 & $229469402-229804452$ & Loss & 11 & EGLNI & Erythrocytosis, familial, 3 \\
\hline & & & & & GNPAT & $\begin{array}{l}\text { Chondrodysplasia punctata, rhizomelic, } \\
\text { type } 2\end{array}$ \\
\hline $31^{a}$ & 3 & $15281603-15545420$ & Gain & 8 & COLQ & Endplate acetylcholinesterase deficiency \\
\hline \multirow[t]{2}{*}{38} & 3 & $|30| 7|| 89-13074|2| \mid$ & Loss & 2 & GPQ & Bernard-Soulier syndrome, type C \\
\hline & & & & & $\mathrm{RHO}$ & Retinitis pigmentosa 4 \\
\hline $40^{a}$ & 3 & |66493869-167384443 & Loss & 4 & $B C H E$ & Apnea, postanesthetic \\
\hline 80 & 5 & $|47335938-| 474607 \mid 6$ & Loss & 2 & SPINK5 & Atopy \\
\hline 111 & 7 & $80115926-80209630$ & Loss & 10 & $C D 36$ & Platelet glycoprotein IV deficiency \\
\hline $120^{a}$ & 7 & $|4203548|-|42| 556 \mid 3$ & Loss & 2 & PRSSI & Trypsinogen deficiency \\
\hline$|2|^{a}$ & 7 & $|5425454|-\mid 55708987$ & Gain & 6 & $\mathrm{SHH}$ & Coloboma, ocular \\
\hline $124^{a}$ & 8 & |30|6678-13270959 & Loss and Gain & 4 & $D L C I$ & Colorectal cancer \\
\hline 147 & 9 & $103072622-103206674$ & Loss & 3 & BAAT & Hypercholanemia, familial \\
\hline \multirow[t]{5}{*}{$149 a$} & 9 & |34892783-137859477 & Gain & 8 & ADAMTSI3 & $\begin{array}{l}\text { Thrombotic thrombocytopenic purpura, } \\
\text { familial }\end{array}$ \\
\hline & & & & & CEL & $\begin{array}{l}\text { Maturity-onset diabetes of the young, type } \\
\text { VIII }\end{array}$ \\
\hline & & & & & COL5AI & Ehlers-Danlos syndrome, type I \\
\hline & & & & & $D B H$ & Dopamine beta-hydroxylase deficiency \\
\hline & & & & & SURFI & Leigh syndrome, due to COX deficiency \\
\hline \multirow[t]{2}{*}{$152^{a}$} & 10 & $49832039-50706976$ & Gain & 5 & CHAT & $\begin{array}{l}\text { Myasthenic syndrome, congenital, } \\
\text { associated with episodic apnea }\end{array}$ \\
\hline & & & & & $E R C C 6$ & Cerebrooculofacioskeletal syndrome I \\
\hline $156^{a}$ & 10 & $|2565| 32|-| 26729328$ & Loss & 2 & $O A T$ & $\begin{array}{l}\text { Gyrate atrophy of choroid and retina with } \\
\text { ornithinemia }\end{array}$ \\
\hline $163^{a}$ & 12 & $2449|8|-34 \mid 0784$ & Gain & 6 & CACNAIC & Timothy syndrome \\
\hline 164 & 12 & $4794562-5039110$ & Gain & 3 & KCNAI & Episodic ataxia/myokymia syndrome \\
\hline \multirow[t]{4}{*}{$167^{a}$} & 12 & $21603812-21931308$ & Loss & 8 & $A B C C 9$ & Cardiomyopathy, dilated, 10 \\
\hline & & & & & GYS2 & Glycogen storage disease, type 0 \\
\hline & & & & & KCNJ8 & Prinzmetal angina \\
\hline & & & & & $L D H B$ & Lactate dehydrogenase-B deficiency \\
\hline \multirow[t]{2}{*}{$176^{a}$} & 13 & $22656237-23918863$ & Gain & 2 & SACS & Spastic ataxia, Charlevoix-Saguenay type \\
\hline & & & & & SGCG & Muscular dystrophy, limb-girdle, type $2 C$ \\
\hline 206 & 16 & $11215600-11869359$ & Loss & 4 & LITAF & Charcot-Marie-Tooth disease, type IC \\
\hline \multirow[t]{6}{*}{$212^{a}$} & 17 & $72497067-78|8| 864$ & Gain & 5 & ACTGI & Deafness, autosomal dominant $20 / 26$ \\
\hline & & & & & ASPSCRI & Alveolar soft-part sarcoma \\
\hline & & & & & FSCN2 & Retinitis pigmentosa-30 \\
\hline & & & & & GAA & Glycogen storage disease II \\
\hline & & & & & SGSH & Sanfilippo syndrome, type A \\
\hline & & & & & SOCS3 & Dermatitis, atopic, 4 \\
\hline \multirow[t]{4}{*}{$215^{a}$} & 18 & $2624 \mid 506-27419753$ & Loss & 5 & DSC2 & $\begin{array}{l}\text { Arrhythmogenic right ventricular } \\
\text { dysplasia, familial, II }\end{array}$ \\
\hline & & & & & DSGI & Keratosis palmoplantaris striata I \\
\hline & & & & & DSG2 & $\begin{array}{l}\text { Arrhythmogenic right ventricular } \\
\text { dysplasia, familial, } 10\end{array}$ \\
\hline & & & & & DSG4 & $\begin{array}{l}\text { Hypotrichosis, localized, autosomal } \\
\text { recessive }\end{array}$ \\
\hline $217^{a}$ & 18 & $5|204895-5| 803567$ & Loss & 4 & TCF4 & Pitt-Hopkins syndrome \\
\hline \multirow[t]{2}{*}{$230^{a}$} & 22 & $47 \mid 87524-49023156$ & Gain & 4 & $A L G / 2$ & $\begin{array}{l}\text { Congenital disorder of glycosylation, type } \\
\text { Ig }\end{array}$ \\
\hline & & & & & $\mathrm{MLCl}$ & $\begin{array}{l}\text { Megalencephalic leukoencephalopathy } \\
\text { with subcortical cysts }\end{array}$ \\
\hline
\end{tabular}

${ }^{a}$ CNVRs reported in the database of genomic variants.

$b$ Total number of gains and losses observed for a CNVR.

c Gene overlapping a CNVR based on ReqSeq of the UCSC March 2006 assembly.

${ }^{d}$ Disease or disease susceptibility associated with the gene, according to the OMIM Morbid Map. 
USA) according to the manufacturer's instructions. The SNP call rate of these samples examined in this study was $98.04 \pm 0.8 \%$.

In addition, the intensity data from $100 \mathrm{~K}$ GeneChip microarrays of 270 individuals were downloaded from the HapMap project website http://www.hapmap.org/; the CNV data for these individuals were collected previously [5]. These individuals included 30 Caucasian trios, 30 African trios and 90 unrelated Asian individuals. The HapMap reference group, which included 210 multi-ethnic unrelated individuals, was used to determine the parameters of CNIT for SNP CN estimation. Forty offspring were used as a training group for the HMM method, and 20 additional offspring were used as a test group to evaluate the FPR and FNR.

\section{Copy Number Inferring Tool}

The CNIT algorithm showed good performance in $\mathrm{CN}$ estimation of SNPs and DNA segments [11], and wellcharacterized HapMap samples were used to determine the parameters for CNIT. In both single-point and multipoint $\mathrm{CN}$ estimations, results obtained from CNIT showed greater accuracy and reduced FPR and FNR compared with that obtained from other programs. When the smoothing procedure was not used, CNIT could detect small CN-altered regions that were missed with other programs. SNPs located in the reported CNV regions of the corresponding individuals were removed, such that the $\mathrm{CN}$ for most of the autosomal SNPs from the reference, training and test groups was two. The $100 \mathrm{~K}$ GeneChip consists of two chips, each with $>50,000$ SNPs $(58,960$ for the XbaI chip and 57,244 for the HindIII chip). The reference group was used to process probe selection, estimate the coefficient of preferential amplification/ hybridization, and construct a $\mathrm{CN}$ distribution for each SNP [11]. After probe selection, 94,587 SNPs (mean intermarker distance $=30.9 \mathrm{~kb}$ ) were used to represent $\mathrm{CN}$ dosage. Finally, EP and transition probabilities of the HMM method were determined using the training and test groups, and were used to identify true $\mathrm{CN}$-altered regions and eliminate false-positive $\mathrm{CN}$ inference by considering contiguous SNPs [11].

\section{CNV validations}

Primer Express Software version 3.0 (Applied Biosystems, Foster City, CA, USA) was used to design PCR primers for the selected target CNVs. Quantitative PCR experiments were performed using the ABI PRISM 7900 Sequence Detector (Applied Biosystems). PCR reactions were performed using the Power SYBR-Green PCR reagent kit (Applied Biosystems) and each reaction mix (25 $\mu$ lotal) contained 2.5 ng genomic DNA for each CNV. qPCR comprised initial denaturation at $94^{\circ} \mathrm{C}$ for 3 minutes, 40 cycles of denaturation at $94^{\circ} \mathrm{C}$ for 15 seconds, and a com- bination of annealing and extension at $60^{\circ} \mathrm{C}$ for 60 seconds. The fluorescence signal was detected in real-time during the qPCR procedure. The primer pair for the long interspersed nuclear elements 1 (LINE1) sequence was used for normalization [18]. The mean estimated CN was calculated from triplicate PCR reactions for each individual.

The Agilent $244 \mathrm{~K}$ CGH array (Agilent Technologies, Palo Alto, CA, USA) contains 236,000 coding and non-coding sequences at a resolution of $6.4 \mathrm{~kb}$. Amplified DNA ( $2 \mu \mathrm{g})$ was used for $\mathrm{Cy} 5 / \mathrm{Cy} 3$ labeling according to the manufacturer's protocol. After array hybridization and scanning, $\mathrm{CN}$-altered regions were identified using CGH analytics 3.4 software (Agilent Technologies) with a threshold zscore of 2.5 .

\section{Statistical analysis}

After CN estimation for each SNP, CN-altered regions were predicted using the HMM package http:// www.cfar.umd.edu/ kanungo/software/software.html based on single-point results. The means of SNP $P$ values and $\mathrm{CN}$ represent the statistical significance and $\mathrm{CN}$ of state-changed segments, respectively. The above data analyses were performed using SAS/STAT version 8 software (SAS Institute, Cary, NC, USA).

\section{Abbreviations}

CGH: comparative genomic hybridization; $\mathrm{CN}$ : copy number; CNIT: copy number inferring tool; CNV: copy number variation; CNVR: copy number-variable region; DBH: dopamine beta-hydroxylase; ECYT3: erythrocytosis type 3; EGLN1: egl nine homolog 1; EP: emission probability; FNR: false-negative rate; FPR: false-positive rate; HMM: hidden Markov model; LINE1: long interspersed nuclear elements 1; miRNA: microRNA; SNP: single nucleotide polymorphism; SD: segmental duplication.

\section{Authors' contributions}

CHL carried out this study and drafted the manuscript. LHL and TPC provided experimental assistance, and SFH, JYW and YTC provided analytic and data assistance. CSJF provided analytic support and supervised the project. All authors have read and approved this final manuscript.

\section{Additional material}

\section{Additional file 1}

Copy number-variable regions (CNVRs) and individual copy number variations (CNVs) in this study. The data contain the list of CNVRs and individual CNVs found in this study.

Click here for file

[http://www.biomedcentral.com/content/supplementary/14712156-9-92-S1.xls] 


\section{Additional file 2}

Genes in the copy number-variable regions (CNVRs). The data contain the genes with variable copy number in this study.

Click here for file

[http://www.biomedcentral.com/content/supplementary/14712156-9-92-S2.xls]

\section{Acknowledgements}

We thank the National Genotyping Center and National Clinical Core, Academia Sinica, Taiwan, for providing DNA samples and genotyping. This project was supported by the National Science Council grant of Taiwan (NSC 95-23/4-B-00I-0I3) and the Institute of Biomedical Sciences,

Academia Sinica, Taiwan. We also thank the two anonymous reviewers for their constructive suggestions, which have largely improved this manuscript.

\section{References}

I. Wright AF: Nature Encyclopedia of the Human Genome. Volume 2. New York: John Wiley \& Sons, Inc.; 2003.

2. Freeman JL, Perry GH, Feuk L, Redon R, McCarroll SA, Altshuler DM, Aburatani $\mathrm{H}$, Jones KW, Tyler-Smith C, Hurles ME, et al.: Copy number variation: new insights in genome diversity. Genome research 2006, I6(8):949-96I.

3. lafrate AJ, Feuk L, Rivera MN, Listewnik ML, Donahoe PK, Qi Y, Scherer SW, Lee C: Detection of large-scale variation in the human genome. Nature genetics 2004, 36(9):949-95I.

4. Jakobsson M, Scholz SW, Scheet P, Gibbs JR, VanLiere JM, Fung HC, Szpiech ZA, Degnan JH, Wang K, Guerreiro R, et al.: Genotype, haplotype and copy-number variation in worldwide human populations. Nature 2008, 45 I (7 I8I):998-1003.

5. Redon R, Ishikawa S, Fitch KR, Feuk L, Perry GH, Andrews TD, Fiegler $\mathrm{H}$, Shapero MH, Carson AR, Chen W, et al.: Global variation in copy number in the human genome. Nature 2006, 444(7 I I 8):444-454.

6. Sebat J, Lakshmi B, Troge J, Alexander J, Young J, Lundin P, Maner S, Massa $\mathrm{H}$, Walker $\mathrm{M}$, Chi M, et al.: Large-scale copy number polymorphism in the human genome. Science 2004, 305(5683): 525-528.

7. Wong KK, deLeeuw RJ, Dosanjh NS, Kimm LR, Cheng Z, Horsman $\mathrm{DE}$, MacAulay C, Ng RT, Brown CJ, Eichler EE, et al: A comprehensive analysis of common copy-number variations in the human genome. American journal of human genetics 2007, 80(I):91-104.

8. Hochstenbach R, Ploos van Amstel HK, Poot M: Microarray-based genome investigation: molecular karyotyping or segmental aneuploidy profiling? Eur J Hum Genet 2006, 14(3):262-265.

9. Huang J, Wei W, Zhang J, Liu G, Bignell GR, Stratton MR, Futreal PA, Wooster R, Jones KW, Shapero MH: Whole genome DNA copy number changes identified by high density oligonucleotide arrays. Human genomics 2004, I(4):287-299.

10. Slater HR, Bailey DK, Ren H, Cao M, Bell K, Nasioulas S, Henke R, Choo KH, Kennedy GC: High-resolution identification of chromosomal abnormalities using oligonucleotide arrays containing 1 16,204 SNPs. American journal of human genetics 2005, 77(5):709-726.

II. Lin CH, Huang MC, Li LH, Wu JY, Chen YT, Fann CS: Genomewide copy number analysis using copy number inferring tool (CNIT) and DNA pooling. Human mutation 2008, 29(8): $1055-1062$.

12. Huang J, Wei W, Chen J, Zhang J, Liu G, Di X, Mei R, Ishikawa S, Aburatani $\mathrm{H}$, Jones $\mathrm{KW}$, et al: CARAT: a novel method for allelic detection of DNA copy number changes using high density oligonucleotide arrays. BMC bioinformatics 2006, 7:83.

13. Oudjeriouat N, Moreau Y, Santimone M, Svensson B, MarchisMouren G, Desseaux V: On the mechanism of alpha-amylase. European journal of biochemistry/FEBS 2003, 270(1 9):387|-3879.

14. Perry GH, Dominy NJ, Claw KG, Lee AS, Fiegler H, Redon R, Werner J, Villanea FA, Mountain JL, Misra R, et al.: Diet and the evolution of human amylase gene copy number variation. Nature genetics 2007, 39(10): 1256-1260.

15. Bartel DP: MicroRNAs: genomics, biogenesis, mechanism, and function. Cell 2004, I | 6(2):28I-297.

16. Pan WH, Fann CS, Wu JY, Hung YT, Ho MS, Tai TH, Chen YJ, Liao C], Yang ML, Cheng AT, et al.: Han Chinese cell and genome bank in Taiwan: purpose, design and ethical considerations. Human heredity 2006, 6I (I):27-30.

17. Yang HC, Liang YJ, Huang MC, Li LH, Lin CH, Wu JY, Chen YT, Fann CS: A genome-wide study of preferential amplification/ hybridization in microarray-based pooled DNA experiments. Nucleic acids research 2006, 34(I5):el06.

18. Li LH, Ho SF, Chen CH, Wei CY, Wong WC, Li LY, Hung Sl, Chung WH, Pan WH, Lee MT, et al.: Long contiguous stretches of homozygosity in the human genome. Human mutation 2006 27(II): IIII5-II2I.

19. Enright AJ, John B, Gaul U, Tuschl T, Sander C, Marks DS: MicroRNA targets in Drosophila. Genome biology 2003, 5(I):RI.
Publish with Bio Med Central and every scientist can read your work free of charge

"BioMed Central will be the most significant development for disseminating the results of biomedical research in our lifetime. "

Sir Paul Nurse, Cancer Research UK

Your research papers will be:

- available free of charge to the entire biomedical community

- peer reviewed and published immediately upon acceptance

- cited in PubMed and archived on PubMed Central

- yours - you keep the copyright

Submit your manuscript here:

http://www.biomedcentral.com/info/publishing_adv.asp 